\title{
28 Research Square \\ Comparison of gastric emptying time after the ingestion of whisky with isocalorically-adjusted glucose solution
}

Tadashi Okabe ( $\nabla$ t-okabe@nms.ac.jp)

Hitachi, Ltd., Hitachinaka General Hospital

Hideo Terashima

Department of Gastroenterological Surgery, Faculty of Medicine, University of Tsukuba

Atsuhiro Sakamoto

Department of Anesthesiology, Nippon Medical School

Research article

Keywords: gastric emptying time, alcohol, ultrasonography, preoperative fasting

Posted Date: November 5th, 2019

DOI: https://doi.org/10.21203/rs.2.16788/v1

License: (1) (i) This work is licensed under a Creative Commons Attribution 4.0 International License.

Read Full License 


\section{Abstract}

Background The gastric emptying of alcoholic beverages remains unclear because the current preoperative fasting guidelines state that clear fluids do not include alcohol, which has led to no information being available on the gastric emptying of alcoholic beverages, and inconsistent findings from previous studies. We demonstrated that liquid gastric emptying mainly depended on energy content, regardless of compositional differences. Therefore, we performed the present study to compare gastric emptying times between whisky mixed with water and sugar water with uniform energy contents and volumes. Methods As a crossover study, 10 healthy male volunteers ingested one of 3 test solutions with a uniform volume of $150 \mathrm{ml}$, i.e., whisky with water-containing whisky $30 \mathrm{ml}(67 \mathrm{kcal})$, sugar water containing glucose $16.8 \mathrm{~g}$ ( $67 \mathrm{kcal})$, and water $(0 \mathrm{kcal})$, and the gastric emptying time of each beverage was then assessed by ultrasound measurements of the gastric antral cross-sectional area. Results The gastric emptying pattern of whisky with water was faster than that of isocaloric sugar water, but slower than that of water. Each antral cross-sectional area 20,30, and 40 min after the ingestion of sugar water was significantly higher than that of whisky with water. Antral cross-sectional areas 10 and 20 min after the ingestion of water were significantly lower than those of whisky with water. Conclusions The results of our study suggested that the gastric emptying time of whisky would be shorter than that of isocaloric glucose solution. Unlike the other beverages, the gastric emptying time of alcohol drinks does not purely depend on the energy content because alcohol itself has no calorie before absorption. The liquid gastric emptying of whisky will theoretically have the similar pattern to that of a calorie-free beverage; however, a slower rate than that of water was observed. Further investigations on the factors contributing to this delay are needed.

\section{Background}

The current American Society of Anesthesiologists (ASA) [1] and European Society of Anaesthesiology (ESA) [2] preoperative fasting guidelines recommend fasting from the intake of clear fluids for 2 hours or more before elective procedures requiring general anaesthesia. Although alcoholic beverages, such as whisky, beer, and wine, do not contain lipids, the ASA preoperative fasting guidelines state that "clear fluids" do not include alcohol; however, the underlying reasons are not stated [1]. The findings of studies on the effects of alcohol on gastric movement and/or emptying are not consistent [3-6], which has led to difficulties with clarifying the gastric emptying of alcoholic beverages on a theoretical basis. Previous studies indicated that the most significant factor influencing liquid gastric emptying was the calorie content of the fluid ingested [7-11]. We elucidate the underlying principle for liquid gastric emptying [7, 11]: 1) gastric volume following the ingestion of liquids decreases exponentially over time; 2 ) under the condition that the initial volume of liquid ingested is fixed, the total amount of calories (calorie content) is a critical factor influencing liquid gastric emptying regardless of compositional differences, e.g., the ratio of fat to carbohydrates; 3 ) when the energy content of the liquid ingested is fixed, the initial volume is a critical factor influencing liquid gastric emptying, which means that the larger the volume, the more rapid the gastric emptying rate in the early phase; 4) The calorie content of the liquid ingested may be the 
primary factor influencing the gastric emptying rate rather than the volume. When this underlying principle is applied to alcoholic beverages, i.e., whisky, the gastric emptying pattern of whisky with water is expected to be closer to that of water when their volumes are uniform; whisky is non-caloric before being absorbed from the gastrointestinal tract because it only includes alcohol and none of the three major nutrients, namely, proteins, fats, and carbohydrates. The alcohol in whiskey only acts as a source of energy equivalent to approximately $7 \mathrm{kcal} / \mathrm{g}$ when being metabolized in the liver [12]. Thus, the aim of the present study is to clarify the gastric emptying pattern of whisky with water by comparison to those of isocalorically-adjusted sugar water or plain water only, and thereby obtain a clearer understanding of the liquid gastric emptying of alcoholic beverages.

\section{Methods}

The present study was registered with the University Hospital Medical Information Network (UMIN) Clinical Trials Registry (ref.: UMIN000034443), and ethical approval was received from the Hitachi Ltd., Hitachi General Hospital Research Ethics Committee (approval number 2018 - 63). This study was designed according to CONSORT guideline.

Ten healthy young male subjects participated in the present study as volunteers. Subjects were limited to males because a previous study showed that gastric emptying was affected by gender, the phase of the menstrual cycle, and the menopausal status [13]. After obtaining written informed consent on the prescribed form凶all 10 subjects were found to be free of medical conditions associated with delayed gastric emptying (e.g., diabetes, severe obesity, and gastric diseases) and alcohol intolerance through a questionnaire that included health history and physical activity. The present study was designed as a crossover study, but was not blinded because the test solution was certainly discriminated by taste.

The study protocol was designed based on the method described in our previous study $[7,11,14]$. According to the recommendations for pre-operative fasting before general anaesthesia $[1,2]$, the ingestion of solids was stopped 6 hours before, and clear fluids 2 hours before the examination. A subject ingested one of the following 3 types of test solutions stored at room temperature over the course of approximately $3 \mathrm{~min}$. After ingesting any one of the 3 types, subjects maintained a seated position at a $45^{\circ}$ angle and underwent an ultrasound examination every $10 \mathrm{~min}$ up to $120 \mathrm{~min}$ after the beverage had been ingested. An ultrasound assessment to calculate the cross-sectional area of the gastric antrum was performed in the right lateral position. In order to ensure technical uniformity, all measurements were performed by only one evaluator (the lead author of this study). The same subject consumed another one of the 3 test solutions on a different day (there was no clearly defined test interval) and underwent the same ultrasound examination. This pattern was repeated until the 3 different test solutions had been ingested in subsequent examinations. The following 3 types of test solutions with a uniform volume were prepared:

1) $150 \mathrm{ml}$ of whisky with water (30 ml of whisky-containing $40 \%$ alcohol $+120 \mathrm{ml}$ of water): Whisky group 
2) $150 \mathrm{ml}$ of sugar water (11.2\% glucose solution): Sugar group

3) $150 \mathrm{ml}$ of water alone: Water group

Whisky with water and that with sugar water were both isocalorically adjusted to $67 \mathrm{kcal}$. In the Whisky group, alcohol intake was equal to that of a so-called "shot of whisky".

In ultrasound imaging, the gastric antrum was identified in the sagittal to right parasagittal plane using the left lobe of the liver, the pancreas, abdominal aorta, or inferior vena cava as anatomical landmarks, as described previously [15-18]. When the minimal antral cross-sectional area of the gastric antrum was imaged using ultrasonography (SonoSite SQ, FUJIFILM SonoSite, Inc., Bothell, WA, USA), the anteroposterior (AP) and craniocaudal (CC) diameters were both measured and recorded in the database, as described previously [15-18].

The following formula was used to calculate the antral cross-sectional area (CSA).

Antral $\mathrm{CSA}=\pi \times \mathrm{AP}(\mathrm{cm}) \times \mathrm{CC}(\mathrm{cm}) / 4$

Gastric volume was calculated from the gastric antral cross-sectional area, as described previously [15].

Gastric volume $=27+14.6 \times \mathrm{CSA}\left(\mathrm{cm}^{2}\right)-1.28 \times$ age $(\mathrm{yr})$

The data obtained as explained above were analysed according to the non-parametric method. A graph of the antral cross-sectional area was made for each group via box-and-whisker plots. A gastric emptying curve was made by plotting the median values of gastric volumes at each measurement point. $\mathrm{A}$ statistical comparison of three groups was performed with the Friedman test. When significant differences were noted, multiple comparisons between the Whisky group and Sugar or Water group were made using the Wilcoxon signed-rank sum test with Bonferroni corrections. A p value of $<0.05$ was considered to be significant. All statistical analyses were conducted using Excel Statistical Program File Ystat 2013 (developed by Yamazaki S, Igakutosyo Syuppan Co., Ltd., Tokyo, Japan).

\section{Results}

Ten healthy male participants consented to, enrolled in, and completed the present study. The following data on their physical characteristics are shown as means and standard deviations (SD); age 32.4 (5.9) years, height $171.2(5.2) \mathrm{cm}$, weight $68.4(8.8) \mathrm{kg}$, and body mass index 23.0 (2.4) kg m². Figure 1 shows the typical ultrasound images obtained during this study.

Using a plot box, Figure 2 shows changes in the gastric antral cross-sectional area over time in each group, i.e., the Whisky group, Sugar group, and Water group. In the Friedman test, significant differences were observed among the groups $(10,20,30$, and 40 min after ingestion; $P<0.01)$. Antral CSA 20,30, and 40 min after ingestion were significantly higher in the Sugar group than in the Whisky group $(P<0.05$; the Wilcoxon signed-rank sum test with Bonferroni corrections; $n=10$; Fig. 2B). Antral CSA 10 and 20 min 
after ingestion were significantly lower in the Water group than in the Whisky group $(P<0.05$; the Wilcoxon signed-rank sum test with Bonferroni corrections; $n=10$; Fig. $2 C$ ). Figure 3 , in which the medians of the calculated gastric volumes in the groups were plotted over time, showed an overview suggesting that the gastric emptying pattern was faster in the Whisky group than in the Sugar group, but slower in the Water group.

\section{Discussion}

Just on the basis of the perspective that the calorie content of an ingested liquid may be the primary factor influencing the gastric emptying rate, the gastric emptying of whisky may have a similar pattern to that of a calorie-free beverage, such as water, because it only includes alcohol with no calories before being absorbed and metabolised. In the present study, the gastric emptying time of whisky with water was shorter than that of the same volume of the isocaloric glucose solution, as anticipated, but was slower than that of the same volume of water, indicating that some factors present in whisky delayed emptying.

Whisky includes only alcohol (ethanol) and none of the three major nutrients, namely, proteins, fats and carbohydrates. Therefore, whisky may be nutritionally deemed a calorie-free beverage before being absorbed from the gastrointestinal tract. Alcohol acts as a source of energy equivalent to approximately $7 \mathrm{kcal} / \mathrm{g}$ only when it is metabolized in the liver. In the liver, alcohol is metabolized in the order of acetaldehyde, acetate, and the end products of water and carbon dioxide. Since acetate derived from this metabolic process is quickly utilized in the tricarboxylic acid cycle, any metabolic products are never stored as an energy source [12]. In contrast, brewed liquors, such as beer and wine, include nutrients that act as an energy source per se, in contrast to alcohol, and, thus, each gastric emptying time is expected to depend on the calorie content. A previous study reported that the gastric emptying time of beer and red wine was significantly longer than that of ethanol under the condition that volumes and alcoholic contents were both uniform, while that of beer, which has a lower calorie content, was shorter than that of wine [6].

Calorie intake is a regulator of the gastric emptying time. Previous studies demonstrated that liquid gastric emptying chiefly depended on the total amount of calories regardless of compositional differences [7-10]. The small intestine chiefly performs digestion and the absorption of water and nutrients, whereas the stomach breaks, stirs, and preserves ingested materials to promote smooth digestion and absorption in the small intestine. If large amounts of ingested nutrients are flowing into the small intestine at once, metabolic abnormalities, including hyperglycaemia, will occur. Dumping syndrome after gastrectomy is one of these pathological conditions. When nutrients are absorbed in the proximal and/or distal small intestine, various gastrointestinal hormones, such as secretin, gastric inhibitory peptide, cholecystokinin, glucagon-like peptides, and peptide YY, are secreted as a feedback mechanism, thereby allowing for the appropriate digestion and absorption of nutrients [19-22], which suppresses the motility of the stomach and upper jejunum. One of those feedback systems is called the “ileal brake” [22]. 
Based on these two standpoints, the gastric emptying pattern of whisky with water is expected be closer to that of water when adjusted to the same volume. In the present study, the gastric emptying time of whisky with water was significantly slower than that of water alone; therefore, the effects of alcohol need to be considered. Although abnormal motility, mucosal damage, and increased permeability occur in the digestive tract following the consumption of alcohol [3], gastric motility exhibits two very different patterns depending on the alcoholic content. Bujanda and co-workers showed that gastric motility was inhibited by the intake of alcoholic beverages with a density of more than $15 \%$, but was promoted at lower densities [4]. Franke and co-workers reported that the gastric emptying times of ethanol adjusted to low alcoholic contents of 4 and $10 \%$ were longer than that of water, and no significant differences were observed in gastric emptying times in the range of $4-10 \%$; however, the underlying mechanisms remain unclear [6]. In the present study, we used whisky with water corresponding to a low alcoholic content of $8 \%$. Although gastric motility is expected to be facilitated by a low alcoholic content, the gastric emptying of whisky with water was significantly longer than that of water in the present study. The following two major factors may be contributing to this phenomenon. The stomach is one of the principal absorption sites of ingested alcohol and has the ability to metabolize ethanol by alcohol dehydrogenase (ADH), such as $A D H 1 C, A D H 3$, and $A D H 4$, despite a low metabolic capacity, suggesting that absorption and metabolic processes in the stomach induced delayed emptying against enhanced gastric motility [23,24]. This insight may be important for the liquid gastric emptying of alcoholic beverages. Furthermore, the osmotic pressure of liquids is a factor that inhibits gastric emptying [25-27]. Previous studies have shown that differences in gastric emptying times due to osmotic pressure were markedly smaller than those due to calorie content [26, 27]; however, a large difference in osmotic pressure significantly affects gastric emptying rates [25]. Since the osmotic pressure of ethanol is $165 \mathrm{mosm} / \mathrm{L}$ per $1 \%$ [6], the osmotic pressure of whisky with alcohol used in the present study was calculated to be at least $1320 \mathrm{mosm} / \mathrm{L}$, which is more than 132 -fold that of water $(10 \mathrm{mosm} / \mathrm{L})$. This large difference results in the slower emptying of whisky with water. Further studies that investigate other factors are required.

There were some limitations in the present study. The number of subjects $(n=10)$ examined was small and all were physically and mentally healthy. Therefore, the results of the present study may not be applied to individuals with alcohol allergies or intolerances or patients including those receiving anaesthesia and undergoing surgery. Furthermore, since the number of subjects $(n=10)$ examined was small, the present study is a type of exploratory study.

\section{Conclusion}

Under the condition of a uniform volume, the gastric emptying time of whisky with water was shorter than that of an isocaloric glucose solution, but longer than that of water. The gastric emptying of whisky did not obey the underlying principle that the calorie content of an ingested liquid is the primary factor influencing the gastric emptying rate, which may be associated with the unique properties of whisky that differ from those of non-alcoholic beverages, i.e., the gastric absorption and metabolism of ethanol and high osmolality. 


\section{Abbreviations}

CSA: cross-sectional area; AP: anteroposterior; CC: craniocaudal; ADH: alcohol dehydrogenase.

\section{Declarations}

\section{Ethics approval and consent to participate}

All participants provided their written informed consent to participate in this study. The study protocol was approved by the Hitachi Ltd., Hitachi General Hospital Research Ethics Committee (approval number $2018-63)$.

\section{Consent for publication}

The present study was registered with the University Hospital Medical Information Network (UMIN) Clinical Trials Registry (ref.: UMIN000034443)

\section{Availability of data and materials}

The data of this study are available from the corresponding author on reasonable request.

\section{Competing interests}

No external funding or competing interests are declared.

\section{Funding}

This study has no financial support.

\section{Authors' contributions}

TO contributed to the study design, conduct of the study, data collection, data analysis, and manuscript preparation. HT contributed to the study design, data analysis, and helped with manuscript preparation. AS gave advice on drafting the manuscript. All authors read and approved the final manuscript.

\section{Acknowledgements}

Not applicable.

\section{Author details}

${ }^{1}$ Department of Anesthesiology, Hitachi, Ltd., Hitachinaka General Hospital, Hitachinaka-shi, Japan. ${ }^{2}$ Visiting Professor, Department of Gastroenterological Surgery, Faculty of Medicine, University of Tsukuba, 
Tsukuba city, Japan. ${ }^{3}$ Professor, Department of Anesthesiology, Nippon Medical School, Bunkyo-ku, Japan.

\section{References}

1. American Society of Anesthesiologists Committee. Practice guidelines for preoperative fasting and the use of pharmacologic agents to reduce the risk of pulmonary aspiration: application to healthy patients undergoing elective procedures: an updated report by the American Society of Anesthesiologists Committee on Standards and Practice Parameters. Anesthesiology. 2011;114(3): 495-511.

2. Smith I, Kranke P, Murat I, Smith A, O'Sullivan G, Søreide E, Spies C, in't Veld B; European Society of Anaesthesiology. Perioperative fasting in adults and children: guidelines from the European Society of Anaesthesiology. Eur J Anaesthesiol. 2011; 28(8): 556-69.

3. Grad S, Abenavoli L, Dumitrascu DL. The Effect of Alcohol on Gastrointestinal Motility. Rev Recent Clin Trials. 2016; 11(3): 191-5.

4. Bujanda L. The effects of alcohol consumption upon the gastrointestinal tract. Am J Gastroenterol. 2000; 95(12): 3374-82.

5. Phaosawasdi K, Tolin R, Mayer E, Fisher RS. Effects of alcohol on the pyloric sphincter. Dig Dis Sci. 1979; 24(12): 934-9.

6. Franke A, Teyssen $\mathrm{S}$, Harder $\mathrm{H}$, Singer MV. Effect of ethanol and some alcoholic beverages on gastric emptying in humans. Scand J Gastroenterol. 2004; 39(7): 638-44.

7. Okabe T, Terashima $\mathrm{H}$, Sakamoto $\mathrm{A}$. What is a determinant of liquid gastric emptying? Comparisons between milk and isocalorically-adjusted clear fluids. Br J Anaesth. 2015; 114(1): 77-82.

8. Brener W, Hendrix TR, McHugh PR. Regulation of the gastric emptying of glucose. Gastroenterology. 1983; 85(1): $76-82$.

9. McHugh PR, Moran TH. Calories and gastric emptying: a regulatory capacity with implications for feeding. Am J Physiol. 1979; 236(5): R254-60.

10. Maughan RJ, Leiper JB, Vist GE. Gastric emptying and fluid availability after ingestion of glucose and soy protein hydrolysate solutions in man. Exp Physiol. 2004; 89(1): 101-8.

11. Okabe T, Terashima $H$, Sakamoto $A$. What is the manner of gastric emptying after ingestion of liquids with differences in the volume under uniform glucose-based energy content? Clin Nutr. 2017; 36(5): 1283-1287.

12. Suter PM. Is alcohol consumption a risk factor for weight gain and obesity? Crit Rev Clin Lab Sci. 2005; 42(3): 197-227.

13. Parkman HP, Harris AD, Miller MA, Fisher RS. Influence of age, gender, and menstrual cycle on the normal electrogastrogram. Am J Gastroenterol. 1996; 91(1): 127-33.

14. Okabe T, Terashima H, Sakamoto A. A comparison of gastric emptying of soluble solid meals and clear fluids matched for volume and energy content: a pilot crossover study. Anaesthesia. 2017; 
72(11): 1344-1350.

15. Van de Putte P, Perlas A. Ultrasound assessment of gastric content and volume. Br J Anaesth. 2014; 113(1): 12-22.

16. Perlas A, Chan VW, Lupu CM, Mitsakakis N, Hanbidge A. Ultrasound assessment of gastric content and volume. Anesthesiology 2009; 111: 82-9.

17. Perlas A, Mitsakakis N, Liu L, et al. Validation of a mathematical model for ultrasound assessment of gastric volume by gastroscopic examination. Anesthesia and Analgesia 2013; 116: 357-63.

18. Hillyard S, Cowman S, Ramasundaram R, Seed PT, O'Sullivan G. Does adding milk to delay gastric emptying? British Journal of Anaesthesia 2014; 112: 66-71.

19. AhIman H, Nilsson O. The gut as the largest endocrine organ in the body. Annals of Oncology 2001; 12: $63-8$.

20. Murphy KG, Bloom SR. Gut hormones and the regulation of energy homeostasis. Nature 2006; $\mathbf{4 4 4}$ : 854-9.

21. Holst JJ. The physiology of glucagon-like peptide 1. Physiological Reviews 2007; 87: 1409-39.

22. Barreto SG, Windsor JA. Does the lleal Brake Contribute to Delayed Gastric Emptying After Pancreatoduodenectomy? Digestive Diseases Sciences 2017; 62: 319-335.

23. Oneta CM, Simanowski UA, Martinez M, et al. First pass metabolism of ethanol is strikingly influenced by the speed of gastric emptying. Gut. 1998; 43: 612-9.

24. Lee SL, Chau GY, Yao CT, et al. Functional assessment of human alcohol dehydrogenase family in ethanol metabolism: significance of first-pass metabolism. Alcoholism: Clinical and Experimental Research 2006; 30: 1132-42.

25. Vist GE, Maughan RJ. The effect of osmolality and carbohydrate content on the rate of gastric emptying of liquids in man. Journal of Physiology 1995; 486: 523-31.

26. Brouns F, Senden J, Beckers EJ, et al. Osmolarity does not affect the gastric emptying rate of oral rehydration solutions. Journal of Parenteral Enteral Nutrition 1995; 19: 403-6.

27. Murray R, Bartoli W, Stofan J, et al. A comparison of the gastric emptying characteristics of selected sports drinks. International Journal of Sport Nutrition 1999; 9: 263-74.

\section{Figures}



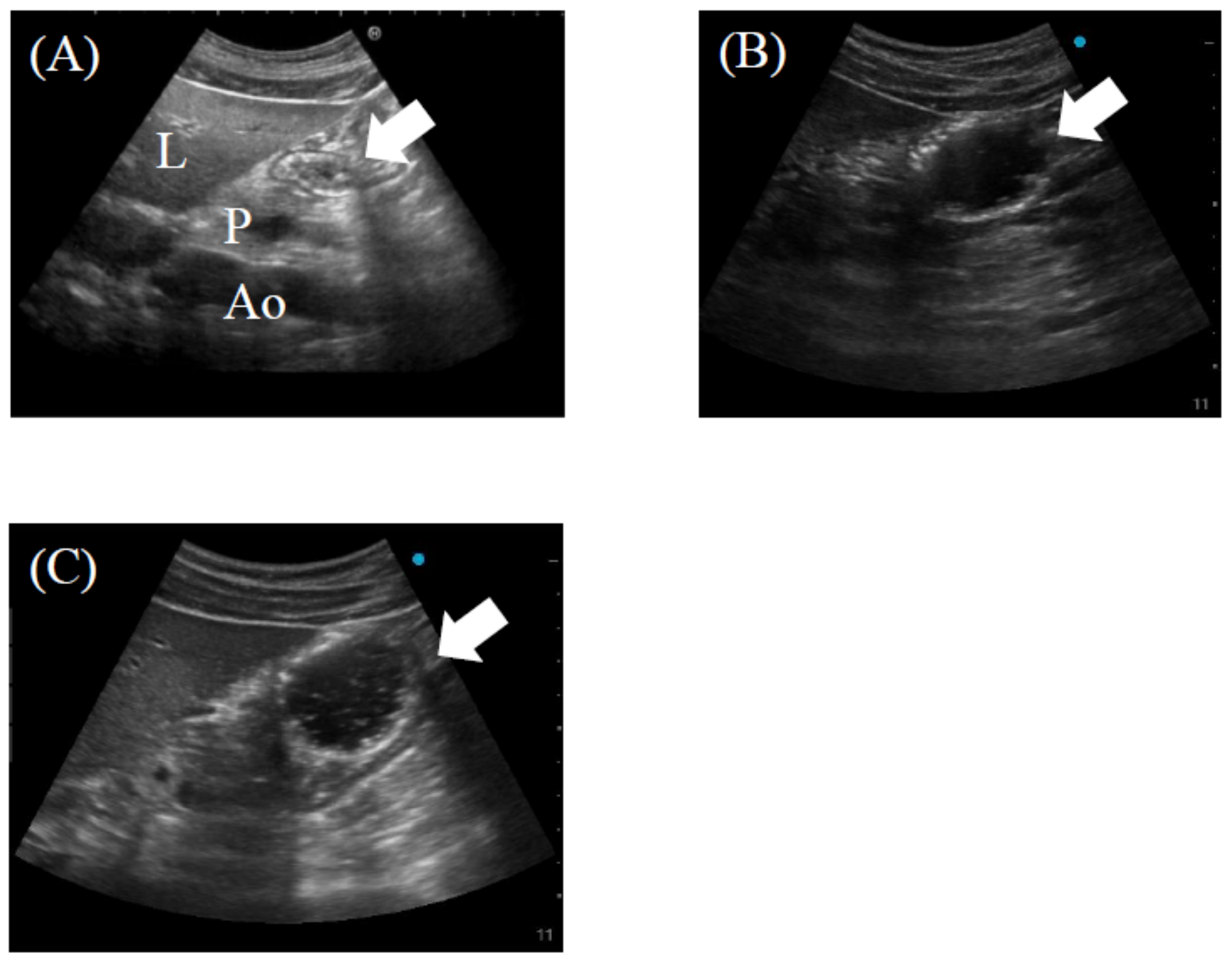

Figure 1

(A) Sagittal sonogram of the empty gastric antrum. The antrum is located between the left lobe of the liver and the pancreas at the level of the aorta or inferior vena cava. (B) Sagittal sonogram of the gastric antrum 10 minutes after the ingestion of $150 \mathrm{ml}$ of diluted whisky ( $30 \mathrm{ml}$ of whisky $+120 \mathrm{ml}$ of water). The antrum appeared distended with a hypoechoic fluid content. (C) Sagittal sonogram of the gastric antrum 10 minutes after the ingestion of $150 \mathrm{ml}$ of $12.5 \%$ glucose solution. The antrum appeared distended with hypoechoic fluid. Arrow = antrum; $\mathrm{L}=$ liver; $\mathrm{P}=$ pancreas; $\mathrm{Ao}=$ aorta 
(A) Whisky group

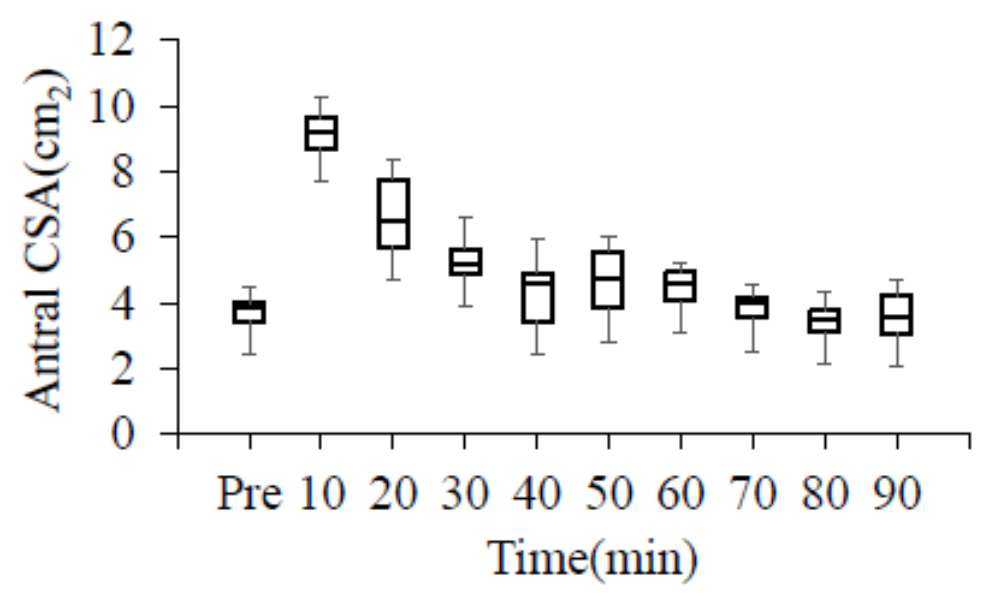

(B) Sugar group

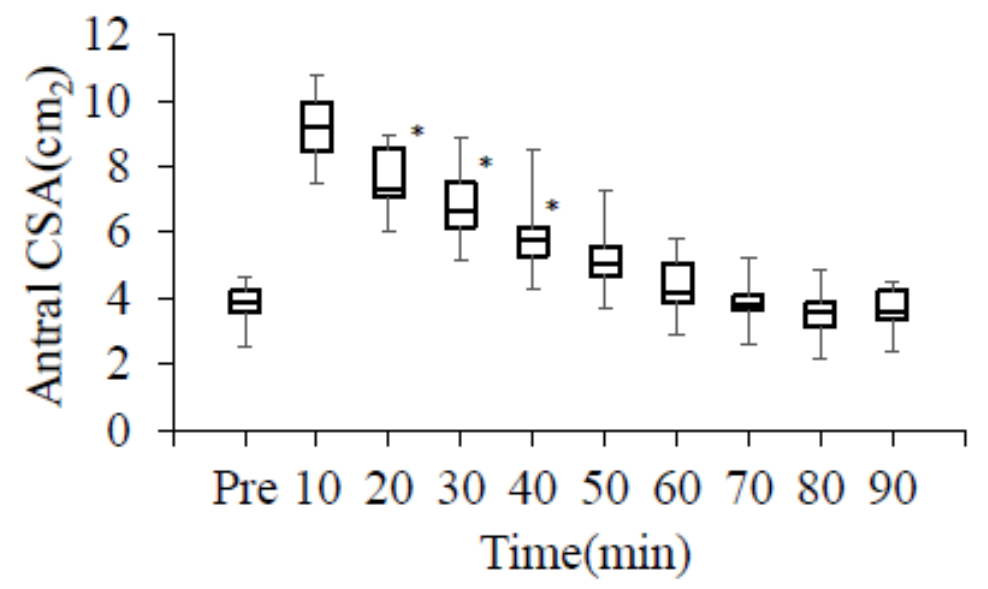

(C) Water group

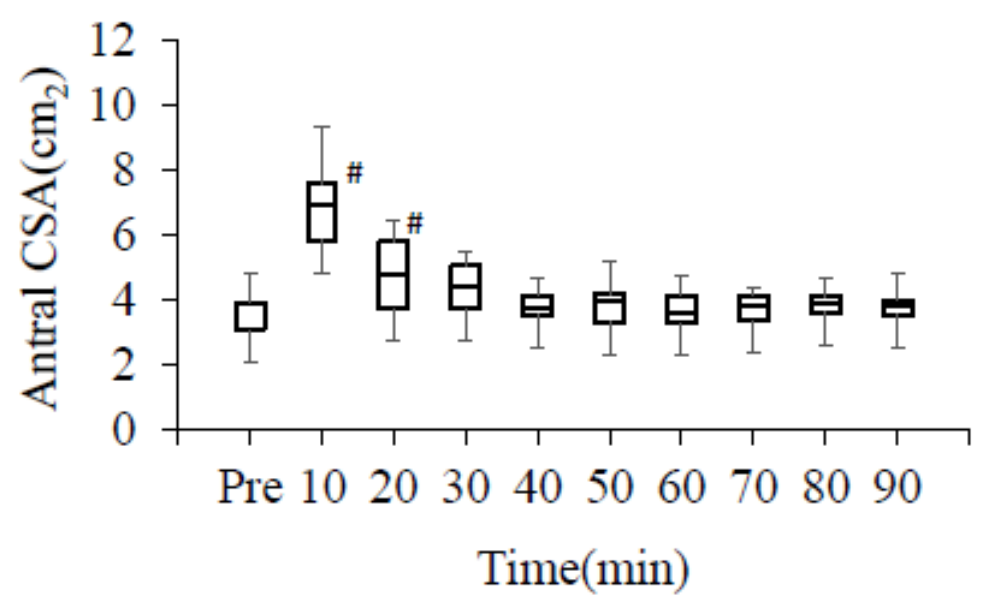

\section{Figure 2}

(A) Changes in gastric antral cross-sectional areas (CSA) over time in the Whisky group. (B) Changes in gastric antral CSA over time in the Sugar group. (C) Changes in gastric antral CSA over time in the Water group. Each data point is presented as a box and whisker plot. The box denotes the first and third quartiles, with the whiskers representing the range. The median is indicated by a line or circle. Whisky group, $150 \mathrm{ml}$ of diluted whisky (30 ml of whisky $+120 \mathrm{ml}$ of water, $67 \mathrm{kcal}$ ); Sugar group, $150 \mathrm{ml}$ of 
$12.5 \%$ glucose solution ( $67 \mathrm{kcal})$; Water group, $150 \mathrm{ml}$ of water $(0 \mathrm{kcal})$. ${ }^{*} \mathrm{P}<0.05$ significant difference between the Whisky group and Sugar group by the Wilcoxon signed-rank sum test with Bonferroni corrections; \#P $<0.05$ significant difference between the Whisky group and Water group by the Wilcoxon signed-rank sum test with Bonferroni corrections.

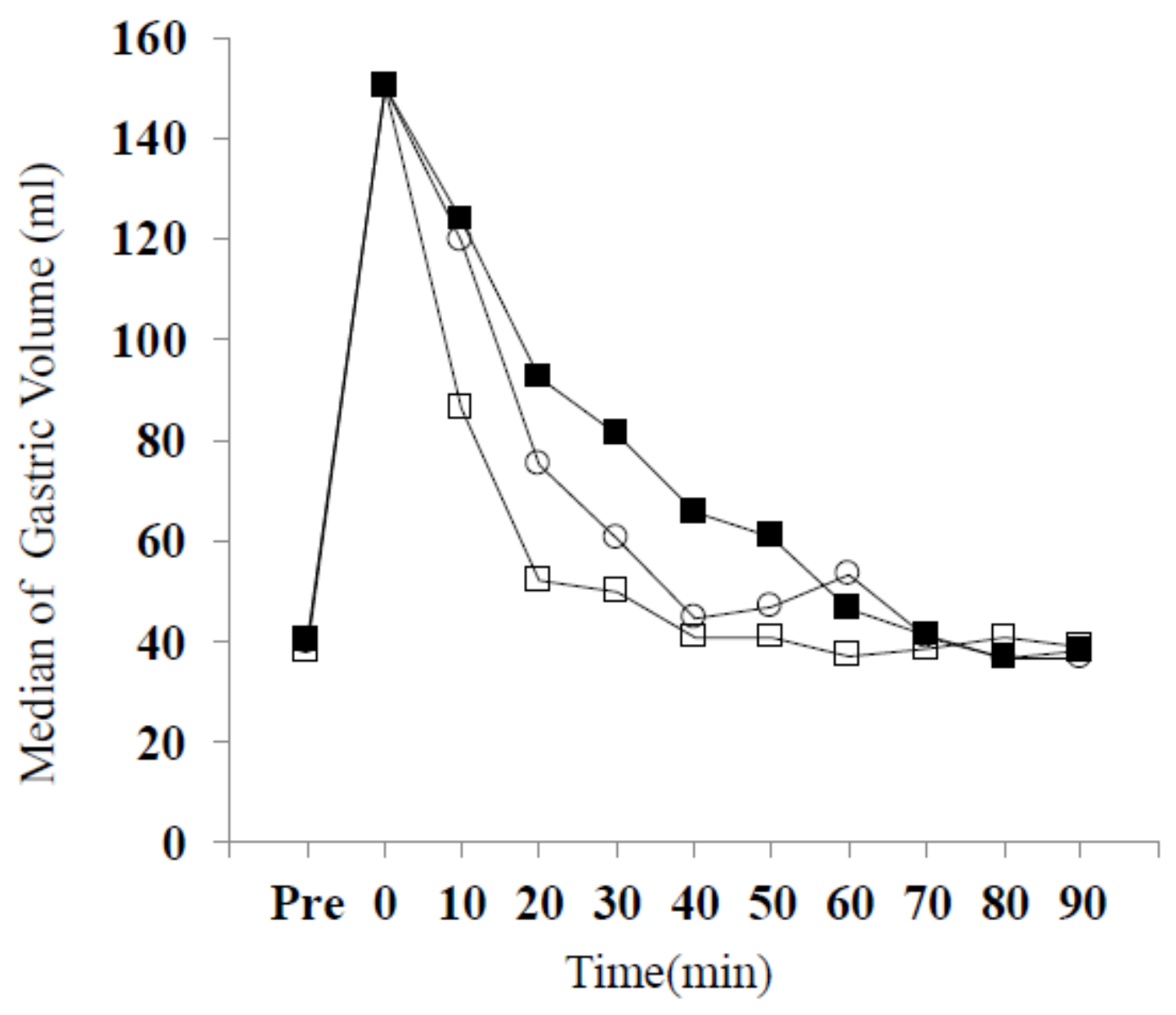

Figure 3

Changes in medians of calculated gastric volumes over time after the ingestion of one of 3 different beverages. Whisky group (), $150 \mathrm{ml}$ of diluted whisky (30 ml of whisky $+120 \mathrm{ml}$ of water, $67 \mathrm{kcal}$ ); Sugar

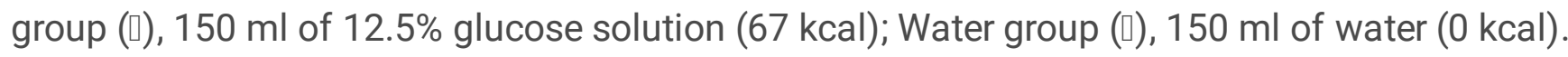

\section{Supplementary Files}

This is a list of supplementary files associated with this preprint. Click to download.

- CONSORT2010Checklist.pdf 